Network Working Group

Request for Comments: 5029

Category: Standards Track
JP. Vasseur

S. Previdi

Cisco Systems, Inc September 2007

\title{
Definition of an IS-IS Link Attribute Sub-TLV
}

Status of This Memo

This document specifies an Internet standards track protocol for the Internet community, and requests discussion and suggestions for improvements. Please refer to the current edition of the "Internet Official Protocol Standards" (STD 1) for the standardization state and status of this protocol. Distribution of this memo is unlimited.

Abstract

This document defines a sub-TLV called "Link-attributes" carried within the TLV 22 and used to flood some link characteristics.

Table of Contents

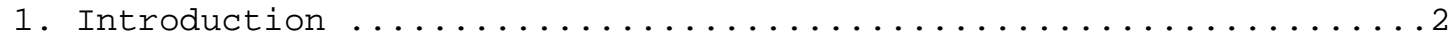

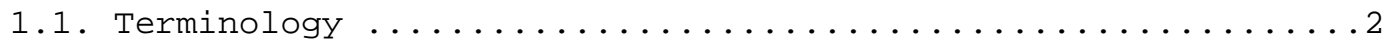

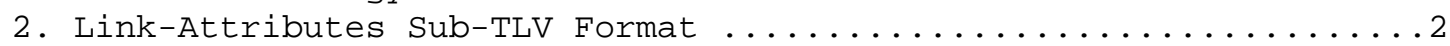

3. Interoperability with Routers Not Supporting This Capability ...3

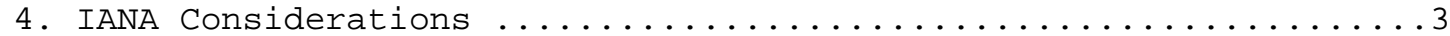

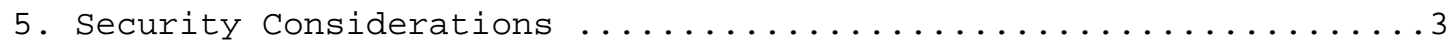

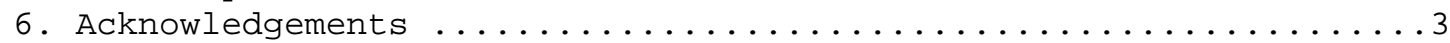

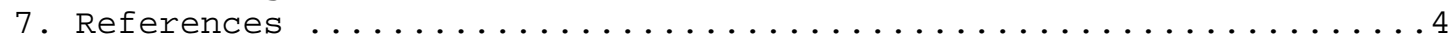

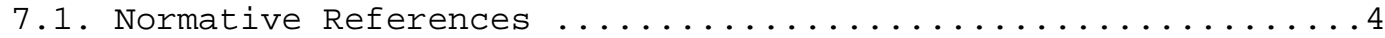

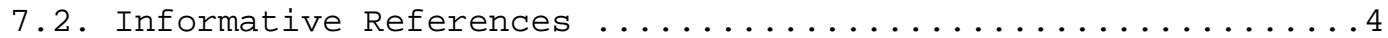




\section{Introduction}

[IS-IS] specifies the IS-IS protocol (ISO 10589) with extensions to support IPv4 in [RFC1195]. A router advertises one or several Link State Protocol data units that are composed of variable length tuples called TLVs (Type-Length-Value).

[RFC3784] defines a set of new TLVs whose aims are to add more information about links characteristics, increase the range of IS-IS metrics, and optimize the encoding of IS-IS prefixes.

This document defines a new sub-TLV named "Link-attributes" carried within the extended IS reachability TLV (type 22) specified in [RFC3784].

\subsection{Terminology}

The key words "MUST", "MUST NOT", "REQUIRED", "SHALL", "SHALL NOT", "SHOULD", "SHOULD NOT", "RECOMMENDED", "MAY", and "OPTIONAL" in this document are to be interpreted as described in RFC 2119 [RFC2119].

\section{Link-Attributes Sub-TLV Format}

The link-attribute sub-TLV is carried within the TLV 22 and has a format identical to the sub-TLV format used by the Traffic Engineering Extensions for IS-IS ([RFC3784]): 1 octet of sub-type, 1 octet of length of the value field of the sub-TLV followed by the value field -- in this case, a 16 bit flags field.

The Link-attribute sub-type is 19 and the link-attribute has a length of 2 octets.

This sub-TLV is OPTIONAL and MUST appear at most once for a single IS neighbor. If a received Link state Packet (LSP) contains more than one Link-Attribute Sub-TLV, an implementation SHOULD decide to consider only the first encountered instance.

The following bits are defined:

Local Protection Available (0x01). When set, this indicates that the link is protected by means of some local protection mechanism (e.g., [RFC4090]).

Link excluded from local protection path (0x02). When set, this link SHOULD not be included in any computation of a repair path by any other router in the routing area. The triggers for setting up this bit are out of the scope of this document. 
3. Interoperability with Routers Not Supporting This Capability

A router not supporting the link-attribute sub-TLV will just silently ignore this sub-TLV.

4. IANA Considerations

IANA has assigned codepoint 19 for the link-attribute sub-TLV defined in this document and carried within TLV 22.

IANA has created a registry for bit values inside the link-attributes sub-TLV. The initial contents of this registry are as follows

$\begin{array}{lll}\text { Value } & \text { Name } & \text { Reference } \\ ---- & ---- & ------- \\ \text { 0x1 } & \text { Local Protection Available } & \text { [RFC5029] } \\ \text { 0x2 } & \text { Link Excluded from Local Protection } & \text { [RFC5029] }\end{array}$

Further values are to be allocated by the Standards Action process defined in [RFC2434], with Early Allocation (defined in [RFC4020]) permitted.

5. Security Considerations

Any new security issues raised by the procedures in this document depend upon the opportunity for LSPs to be snooped and modified, the ease/difficulty of which has not been altered. As the LSPs may now contain additional information regarding router capabilities, this new information would also become available to an attacker. Specifications based on this mechanism need to describe the security considerations around the disclosure and modification of their information. Note that an integrity mechanism, such as one defined in [RFC3567], should be applied if there is high risk resulting from the modification of capability information.

6. Acknowledgements

The authors would like to thank Mike Shand, Les Ginsberg, and Bill Fenner for their useful comments. 
7. References

\subsection{Normative References}

[IS-IS] "Intermediate System to Intermediate System Intra-Domain Routing Exchange Protocol for use in Conjunction with the Protocol for Providing the Connectionless-mode Network Service (ISO 8473)", ISO 10589.

[RFC1195] Callon, R., "Use of OSI IS-IS for routing in TCP/IP and dual environments", RFC 1195, December 1990.

[RFC2119] Bradner, S., "Key words for use in RFCs to Indicate Requirement Levels", BCP 14, RFC 2119, March 1997.

[RFC2434] Narten, T. and H. Alvestrand, "Guidelines for Writing an IANA Considerations section in RFCs", BCP 26, RFC 2434, October 1998 .

[RFC3784] Smit, H. and T. Li, "Intermediate system to Intermediate System (IS-IS) Extensions for Traffic Engineering (TE)", RFC 3784, June 2004 .

[RFC4020] Kompella, K. and A. Zinin, "Early IANA Allocation of Standards Track Code Points", BCP 100, RFC 4020, February 2005 .

7.2. Informative References

[RFC3567] Li, T. and R. Atkinson, "Intermediate System to Intermediate System (IS-IS) Cryptographic Authentication", RFC 3567, July 2003.

[RFC4090] Pan, P., Swallow, G., and A. Atlas, "Fast Reroute Extensions to RSVP-TE for LSP Tunnels", RFC 4090, May 2005. 
Authors' Addresses

JP Vasseur

Cisco Systems, Inc

1414 Massachusetts Avenue

Boxborough, MA 01719

USA

EMail: jpvecisco.com

Stefano Previdi

Cisco Systems, Inc

Via Del Serafico 200

Roma 00142

Italy

EMail: sprevididcisco.com 
Full Copyright statement

Copyright (C) The IETF Trust (2007).

This document is subject to the rights, licenses and restrictions contained in BCP 78, and except as set forth therein, the authors retain all their rights.

This document and the information contained herein are provided on an "AS IS" basis and THE CONTRIBUTOR, THE ORGANIZATION HE/SHE REPRESENTS OR IS SPONSORED BY (IF ANY), THE INTERNET SOCIETY, THE IETF TRUST AND THE INTERNET ENGINEERING TASK FORCE DISCLAIM ALL WARRANTIES, EXPRESS OR IMPLIED, INCLUDING BUT NOT LIMITED TO ANY WARRANTY THAT THE USE OF THE INFORMATION HEREIN WILL NOT INFRINGE ANY RIGHTS OR ANY IMPLIED WARRANTIES OF MERCHANTABILITY OR FITNESS FOR A PARTICULAR PURPOSE.

Intellectual Property

The IETF takes no position regarding the validity or scope of any Intellectual Property Rights or other rights that might be claimed to pertain to the implementation or use of the technology described in this document or the extent to which any license under such rights might or might not be available; nor does it represent that it has made any independent effort to identify any such rights. Information on the procedures with respect to rights in RFC documents can be found in BCP 78 and BCP 79 .

Copies of IPR disclosures made to the IETF Secretariat and any assurances of licenses to be made available, or the result of an attempt made to obtain a general license or permission for the use of such proprietary rights by implementers or users of this specification can be obtained from the IETF on-line IPR repository at http://www.ietf.org/ipr.

The IETF invites any interested party to bring to its attention any copyrights, patents or patent applications, or other proprietary rights that may cover technology that may be required to implement this standard. Please address the information to the IETF at ietf-ipreietf.org. 